\title{
Association of sublingual microcirculation parameters and endothelial glycocalyx dimensions in resuscitated sepsis
}

\author{
Alexandros Rovas ${ }^{1}$, Laura Mareen Seidel ${ }^{2}$, Hans Vink ${ }^{3}$, Timo Pohlkötter ${ }^{1}$, Hermann Pavenstädt ${ }^{1}$, Christian Ertmer ${ }^{2}$, \\ Michael Hessler ${ }^{2+}$ and Philipp Kümpers ${ }^{1 *+}$ (D)
}

\begin{abstract}
Background: The endothelial glycocalyx (eGC) covers the luminal surface of the vascular endothelium and plays an important protective role in systemic inflammatory states and particularly in sepsis. Its breakdown leads to capillary leak and organ dysfunction. Moreover, sepsis-induced alterations of sublingual microcirculation are associated with a worse clinical outcome. The present study was performed to investigate the associations between eGC dimensions and established parameters of microcirculation dysfunction in sepsis.

Methods: This observational, prospective, cross-sectional study included 40 participants, of which 30 critically ill septic patients were recruited from intensive care units of a university hospital and 10 healthy volunteers served as controls. The established microcirculation parameters were obtained sublingually and analyzed according to the current recommendations. In addition, the perfused boundary region (PBR), an inverse parameter of the eGC dimensions, was measured sublingually, using novel data acquisition and analysis software $\left(G l y c o C h e c k^{T M}\right)$. Moreover, we exposed living endothelial cells to 5\% serum from a subgroup of study participants, and the delta eGC breakdown, measured with atomic force microscopy (AFM), was correlated with the paired PBR values.

Results: In septic patients, sublingual microcirculation was impaired, as indicated by a reduced microvascular flow index (MFI) and a reduced proportion of perfused vessels (PPV) compared to those in healthy controls (MFI, 2.93 vs $2.74, p=0.002 ;$ PPV, 98.53 vs $92.58, p=0.0004$ ). PBR values were significantly higher in septic patients compared to those in healthy controls, indicating damage of the eGC $(2.04$ vs $2.34, p<0.0001)$. The in vitro AFM data correlated exceptionally well with paired PBR values obtained at the bedside ( $r s=-0.94, p=0.02$ ). Both PBR values and microcirculation parameters correlated well with the markers of critical illness. Interestingly, no association was observed between the PBR values and established microcirculation parameters.
\end{abstract}

Conclusion: Our findings suggest that eGC damage can occur independently of microcirculatory impairment as measured by classical consensus parameters. Further studies in critically ill patients are needed to unravel the relationship of glycocalyx damage and microvascular impairment, as well as their prognostic and therapeutic importance in sepsis.

Trial registration: Retrospectively registered: Clinicaltrials.gov, NCT03960307

Keywords: Endothelial glycocalyx, Microcirculation, Perfused boundary region, PBR, Intravital microscopy, Sepsis, Intensive care unit, Sidestream dark field microscopy, Incident dark field illumination imaging, Glycosaminoglycans

\footnotetext{
* Correspondence: philipp.kuempers@ukmuenster.de

${ }^{\dagger}$ Michael Hessler and Philipp Kümpers contributed equally and are both considered senior authors.

${ }^{1}$ Department of Medicine D, Division of General Internal Medicine,

Nephrology, and Rheumatology, University Hospital Muenster,

Albert-Schweitzer-Campus 1, 48149 Münster, Germany

Full list of author information is available at the end of the article
}

(c) The Author(s). 2019 Open Access This article is distributed under the terms of the Creative Commons Attribution 4.0 International License (http://creativecommons.org/licenses/by/4.0/), which permits unrestricted use, distribution, and reproduction in any medium, provided you give appropriate credit to the original author(s) and the source, provide a link to the Creative Commons license, and indicate if changes were made. The Creative Commons Public Domain Dedication waiver (http://creativecommons.org/publicdomain/zero/1.0/) applies to the data made available in this article, unless otherwise stated. 


\section{Background}

The endothelial glycocalyx (eGC) is a negatively charged, carbohydrate-rich layer, lining the luminal surface of the entire vascular endothelium $[1,2]$. It is up to $3 \mu \mathrm{m}$ thick, largely consists of highly sulfated glycosaminoglycans and proteoglycans, and plays a pivotal role in the maintenance of microcirculatory homeostasis $[3,4]$. An intact eGC controls capillary permeability, reduces leukocyte-endothelial interactions, mediates shear-induced nitric oxide release, contributes to the regulation of the endothelial redox state, and harbors important anticoagulant mediators [5-7]. Accordingly, the critical importance of the eGC has been highlighted in different vascular pathologies, and particularly in the systemic inflammatory response syndrome (SIRS) and sepsis, where glycocalyx degradation plays a causative role in vascular barrier dysfunction and the development of organ failure, especially lung and kidney injury [8-10].

Observational studies in critically ill patients have shown that the amount of shed eGC constituents in blood samples correlates with sepsis severity and outcome [11-15]. Recently, novel automated acquisition and analysis software (GlycoCheck ${ }^{\mathrm{TM}}$, Microvascular Health Solutions Inc., Salt Lake City, UT, USA), able to analyze the perfused boundary region (PBR), an inverse parameter of eGC dimensions in sublingual microvessels, has become available [16]. Pilot studies conducted in the intensive care unit (ICU) revealed that the PBR is indeed markedly increased in critically ill patients compared to healthy controls $[3,17-20]$. We were recently able to confirm excellent inter- and intra-observer reproducibility of the GlycoCheck ${ }^{\mathrm{TM}}$ method under routine clinical conditions in the emergency room (ER) and ICU [21].

Analysis of the sublingual microcirculation by sidestream dark field (SDF) imaging and incident dark field (IDF) illumination imaging has already been recognized as an interesting tool to improve risk stratification and prognostication and possibly to guide individual therapy in the future [22-27]. For example, De Backer et al. [28] found that the proportion of perfused small sublingual vessels outperformed global hemodynamic variables in the prediction of ICU mortality in patients with severe sepsis. However, whether eGC changes coincide with established parameters of microcirculatory dysfunction in septic patients has not been yet investigated. We therefore measured in vivo, for the first time, eGC dimensions and classical microcirculation parameters simultaneously in septic patients. Additionally, we performed further in vitro measurements to evaluate the accuracy of the calculated in vivo PBR values.

\section{Methods}

\section{Study population and study design}

This prospective, observational, cross-sectional study took place from July 2017 to September 2017 in the medical and surgical ICUs of the University Hospital Münster (>70 ICU beds). The study was performed in accordance with the Declaration of Helsinki, was approved by the competent ethics committee (2016073-f-S), and was retrospectively registered in Clinicaltrials.gov (NCT03960307).

Thirty adult ICU septic patients, as defined by the Sepsis-3 criteria published by the ESICM-SCCM Sepsis Redefinitions Task Force [29], were enrolled non-consecutively after the initial resuscitation. Written informed consent was obtained from the patients or their legal representatives. Exclusion criteria were underage, pregnancy, oral mucosal inflammation, or injury, which could locally influence the sublingual microvasculature. Ten apparently healthy volunteers served as controls.

Demographic variables, routine chemistry tests, and physiological parameters, including the Sequential Organ Failure Assessment (SOFA) score [29] and a contemporary version of the Charlson Comorbidity Index (CCI) [30], were obtained for each subject immediately before the sublingual videomicroscopy (Table 1). Serum samples from patients and controls were obtained and immediately centrifuged at $4{ }^{\circ} \mathrm{C}$ with $4000 \mathrm{~g}$ for $10 \mathrm{~min}$ and stored at $-80^{\circ} \mathrm{C}$ for further analysis of the glycocalyx components.

The subsequent assessment of the microcirculation and the eGC was independently performed in random order by two physicians (AR, LMS), as described in detail below. Both physicians were experienced in these techniques and trained to recognize and avoid pressure and movement artifacts. The real-time assessment of microvascular flow index (MFI by "eyeballing") was used as a post hoc verification to ensure representative recordings of the microvasculature [31]. The different parameters assessed by the two methods are summarized in Additional file 1: Table S1.

\section{Assessment of the endothelial glycocalyx in vivo}

The endothelial glycocalyx was assessed sublingually using the GlycoCheck ${ }^{\text {Tix }}$ software, coupled with a stroboscopic, SDF camera (CapiScope HVCS, KK Technology, Honiton, UK) by a physician experienced in the method (AR).

The software automatically detects, records, and analyzes the microvessels with diameters between 5 and $25 \mu \mathrm{m}$. Specifically, it calculates the dynamic lateral movement of the red blood cells (RBCs) into the permeable part of the eGC layer, expressed as the PBR (in $\mu \mathrm{m}$ ). An impaired eGC permits a greater number of RBCs to 
Table 1 Baseline characteristics

\begin{tabular}{|c|c|c|c|}
\hline Variable & Healthy individuals & Septic patients & $p$ value \\
\hline Number of participants ( $n(\%))$ & 10 & 30 & - \\
\hline Female sex $(n(\%))$ & $5(50)$ & $7(23)$ & 0.11 \\
\hline Age (years, median (IQR)) & $30(27-34)$ & $67(58-80)$ & $<0.0001$ \\
\hline $\mathrm{BMI}\left(\mathrm{kg} / \mathrm{m}^{2}\right.$, median $\left.(\mathrm{IQR})\right)$ & $23.6(21.3-26.1)$ & $25.3(21.7-28.2)$ & 0.34 \\
\hline Duration of sepsis at study inclusion (days, median $(\mathrm{IQR}))^{*}$ & - & $2(1-5)$ & - \\
\hline SOFA score (median (IQR)) & - & $9(5-12)$ & - \\
\hline Organ replacement therapy (n (\%)) & - & $18(60)$ & - \\
\hline Mechanical ventilation ( $n(\%))$ & - & $17(56.7)$ & - \\
\hline Acute dialysis ( $n(\%))$ & - & $5(16.7)$ & - \\
\hline Vasopressors (n (\%)) & - & $20(66.7)$ & - \\
\hline Norepinephrine dose ( $\mu \mathrm{g} / \mathrm{kg} / \mathrm{min})$ & - & $0.05(0-0.16)$ & - \\
\hline Septic shock $(n(\%))^{* *}$ & - & $3(10)$ & - \\
\hline Hospital mortality (n (\%)) & - & $10(33.3)$ & - \\
\hline CCl score (median (IQR)) & - & $1.5(0.8-2.3)$ & - \\
\hline \multicolumn{4}{|l|}{ Comorbidities (n (\%)) } \\
\hline Chronic respiratory disease & - & $8(26.7)$ & - \\
\hline Congestive heart failure & - & $16(53.3)$ & - \\
\hline Chronic hepatic disease & - & $3(10)$ & - \\
\hline Dialysis-dependent CKD & - & $1(3.3)$ & - \\
\hline Malignancy & - & $5(16.7)$ & - \\
\hline Diabetes mellitus & - & $5(16.7)$ & - \\
\hline \multicolumn{4}{|l|}{ Focus of infection ( $n(\%))$} \\
\hline Respiratory tract & - & $17(56.7)$ & - \\
\hline Prosthesis & - & $4(13.3)$ & - \\
\hline Gastrointestinal tract & - & $3(10)$ & - \\
\hline Unknown & - & $2(6.7)$ & - \\
\hline Skin & - & $2(6.7)$ & - \\
\hline Urinary tract & - & $1(3.3)$ & - \\
\hline Heart & - & $1(3.3)$ & - \\
\hline \multicolumn{4}{|l|}{ Endothelial glycocalyx (median (IQR)) } \\
\hline PBR 5-25 ( $\mu \mathrm{m})$ & $2.04(1.97-2.10)$ & $2.34(2.21-2.46)$ & $<0.0001$ \\
\hline Syndecan-1 (ng/ml) & $21.3(13.2-56.7)$ & 204.5 (114.2-358.9) & $<0.0001$ \\
\hline \multicolumn{4}{|l|}{ Microcirculation data (median (IQR)) } \\
\hline $\operatorname{TVD}\left(\mathrm{mm} / \mathrm{mm}^{2}\right)$ & $18.88(17.56-21.68)$ & $19.17(17.06-20.24)$ & 0.87 \\
\hline $\mathrm{PVD}\left(\mathrm{mm} / \mathrm{mm}^{2}\right)$ & $18.54(17.19-21.26)$ & $16.97(14.96-19.87)$ & 0.08 \\
\hline PPV (\%) & $98.73(96.55-99.80)$ & $92.58(85.63-97.14)$ & 0.0004 \\
\hline MFI (points) & $2.93(2.89-2.96)$ & $2.74(2.58-2.91)$ & 0.002 \\
\hline HI (\%) & $0.04(0.01-0.06)$ & $0.08(0.03-0.16)$ & 0.04 \\
\hline \multicolumn{4}{|l|}{ Macrocirculation data (median (IQR)) } \\
\hline MAP (mmHg) & $94.2(85.4-102.4)$ & $73.0(67.5-84.6)$ & 0.0001 \\
\hline Heart rate (pulse/min) & $73(65-83)$ & $91(81-101)$ & 0.0004 \\
\hline Respiratory rate (breaths/min) & $14(13-15)$ & $20(17-26)$ & $<0.0001$ \\
\hline Temperature $\left({ }^{\circ} \mathrm{C}\right)$ & $36.6(36.5-36.8)$ & $37(36.4-37.7)$ & 0.12 \\
\hline
\end{tabular}

Laboratory data (median (IQR)) 
Table 1 Baseline characteristics (Continued)

\begin{tabular}{|c|c|c|c|}
\hline Variable & Healthy individuals & Septic patients & $p$ value \\
\hline CRP $(\mathrm{mg} / \mathrm{dl})$ & 0.5 & $23.2(16.9-33.3)$ & $<0.0001$ \\
\hline IL-6 (ng/ml) & $2.0(2.0-2.5)$ & $367.0(96.0-1121.0)$ & $<0.0001$ \\
\hline PCT (ng/ml) & $0.04(0.03-0.05)$ & $9.08(1.24-48.23)$ & $<0.0001$ \\
\hline $\mathrm{pH}$ & - & $7.42(7.36-7.48)$ & - \\
\hline Lactate (mmol/l) & $0.90(0.65-1.20)$ & $1.70(0.98-2.00)$ & 0.001 \\
\hline Albumin (g/dl) & $4.8(4.5-5.0)$ & $2.5(2.0-3.0)$ & $<0.0001$ \\
\hline Total serum protein (g/dl) & $7.1(6.8-7.5)$ & $5.5(5.1-6.0)$ & $<0.0001$ \\
\hline
\end{tabular}

$p$ value was calculated between healthy individuals and ICU patients

$B M I$ body mass index, $C C l$ score Charlson Comorbidity Index score, $C K D$ chronic kidney disease, $C N S$ central nervous system, $C R P C$-reactive protein, $H I$

heterogeneity index, IL-6 interleukin-6, IQR interquartile range, MAP mean arterial pressure, MFI microvascular flow index, $P B R$ perfused boundary region, $P C T$

procalcitonin, PPV proportion of perfused vessels, PVD perfused vessel density, RBC red blood cell, SOFA score Sequential Organ Failure Assessment score, TVD

total vessel density, WBC white blood cell

${ }^{*} n=9$ of 30 patients (30\%) were included within $24 \mathrm{~h}$ after fulfilling Sepsis- 3 criteria

**Septic shock: vasopressors required to maintain MAP $\geq 65 \mathrm{mmHg}$ and serum lactate $>2 \mathrm{mmol} / \mathrm{l}$.

penetrate deep into the endothelium, which is translated as an increase in the PBR value [16].

Briefly, the GlycoCheck ${ }^{\text {tw }}$ software allows video acquisition after predefined image quality criteria (motion, intensity, and focus) are fulfilled. Each complete measurement consists of at least 10 5-s videos (40 frames/ video), containing a total of about 3000 vascular segments of $10 \mu \mathrm{m}$ each. All videos are deliberately obtained from different positions in the sublingual microvasculature. The software automatically subjects the vascular segments obtained to a strict quality check. After marking and discarding invalid segments, the software obtains up to 840 radial intensity profiles for each valid vascular segment and, based on the presence of RBCs, calculates the RBC filling percentage, signal quality, and RBC column width (RBCW). The distribution of RBCs in each valid segment defines the median $\mathrm{RBCW}$, as well as the outer edge of the RBC-perfused lumen (Dperf). The PBR is defined as the distance between the RBCW and the outer edge of the Dperf and is calculated using the following formula: (Dperf - RBCW)/ 2. The software classifies the PBR values to their corresponding RBCW $(5-25 \mu \mathrm{m})$, presents a median PBR for each vessel diameter category $(5-9 \mu \mathrm{m}, 10-19 \mu \mathrm{m}$, and $20-25 \mu \mathrm{m}$ ), and provides a single, average, weighted PBR value $(5-25 \mu \mathrm{m})$ for each measurement. Data from two complete measurements (hereafter referred to as "measurement set") were manually averaged to avoid sampling error and to counterbalance spatial heterogeneity of the sublingual microcirculation. The excellent interand intra-observer reproducibility under real-life conditions has been previously reported by our group [21].

\section{Assessment of sublingual microcirculation}

The microcirculation was visualized sublingually with the use of an IDF illumination hand-held vital microscope $\left(\right.$ CytoCam $^{\text {sx }}$, Braedius Medical BV, Huizen, The
Netherlands) by a physician experienced in the method (LMS) [32]. At least five videos of the sublingual microcirculation were recorded from different positions in the sublingual region. The videos obtained went through a quality check, based on the recommendations of Massey et al. [33], and were manually discarded if necessary. The remaining 116 high-quality videos were semi-manually analyzed offline by an experienced operator (MH) blinded for the patients' clinical data, as thoroughly described previously [34], using dedicated software (Capillary Mapper 1.4, University of Muenster Medical Centre, Münster, Germany [35]). Total vessel density (TVD), perfused vessel density (PVD), proportion of perfused vessels (PPV), microvascular flow index (MFI), and heterogeneity index (HI) were calculated in the microvessels under $20 \mu \mathrm{m}$, based on current recommendations, in order to assess microcirculation abnormalities in critically ill patients [25], as described in detail previously [36].

\section{Quantification of circulating syndecan-1 levels}

The proteoglycan syndecan-1 (syn-1), an important core protein of the eGC, was measured in serum using a commercially available enzyme-linked immunosorbent assay (ELISA) (Gen-Probe Diaclone Research, Besançon, France). The measurements were performed in duplicate according to the manufacturer's instructions and, at the same time, by the same investigator (AR), blinded to individuals' characteristics.

\section{Assessment of endothelial glycocalyx in vitro \\ Cell culture}

The human umbilical vein endothelial cell (EC) line EA.hy926 was grown on glass coverslips (Roche Diagnostics $\mathrm{GmbH}$, Mannheim, Germany) for at least 3 days until confluence in Dulbecco's modified Eagle's medium (DMEM, Invitrogen, Karlsruhe, Germany) supplemented 
with $10 \%$ fetal bovine serum (FBS, FBS Superior Biochrom, Berlin, Germany) and 1\% penicillin/streptomycin (Biochrom, Berlin, Germany) in a $5 \% \mathrm{CO}_{2}$-enriched environment at $37^{\circ} \mathrm{C}$.

\section{Atomic force microscopy}

The thickness of the eGC in vitro was determined using the atomic force microscope (AFM) nanoindentation technique, as described previously in detail [37-39]. Briefly, cells were analyzed in 4-(2-hydroxyethyl)-1piperazineethanesulfonic acid (HEPES) buffer $(140 \mathrm{mM}$ $\mathrm{NaCl}, 5 \mathrm{mM} \mathrm{KCl}, 1 \mathrm{mM} \mathrm{CaCl} 2,1 \mathrm{mM} \mathrm{MgCl} 2,5 \mathrm{mM}$ glucose, $10 \mathrm{mM}$ HEPES) supplemented with $1 \%$ FBS at $37^{\circ} \mathrm{C}$ in a fluid chamber with a Nanoscope III Multimode AFM (Veeco, Mannheim, Germany). A triangular cantilever (Novascan Technologies, Boone, IA, USA) with a mounted spherical tip (diameter $10 \mu \mathrm{m}$ ) and a spring constant of $10 \mathrm{pN} / \mathrm{nm}$ periodically indents the cells. A laser beam is used to quantify the cantilever deflection. Once the force acting on the cantilever, the piezo displacement, and the deflection sensitivity are known, the thickness of the eGC can be calculated. A more detailed description of the AFM method is provided in Additional file 1: Figure S1.

\section{Statistical analysis}

Data are presented as absolute numbers, percentages, means with standard deviations, or medians with corresponding 25th and 75th percentiles (interquartile range (IQR)), as appropriate. The non-parametric Mann-Whitney $U$ test and the chi-square test were used to compare the parameters between the groups. Agreement between MFIs obtained by eyeballing was visualized by the Bland-Altman method. Spearman rank correlation coefficient was used to assess the correlations between variables. Associations between PBR and microcirculatory parameters were evaluated using multiple linear regression. All the tests used were two-sided, and statistical significance was set at $p<0.05$. Our study was powered to detect a moderate correlation (Spearman correlation coefficient $=0.5$ ) between PBR and the microcirculation parameters in the septic cohort with $80 \%$ power given a two-sided alpha of 0.05 [40]. SPSS version 24 (IBM Corporation, Armonk, NY, USA) and GraphPad Prism version 7 (GraphPad Prism Software Inc., San Diego, CA, USA) were used for statistical analyses and preparation of figures.

\section{Results}

The clinical and demographic characteristics of the 40 study participants are shown in Table 1. Approximately $55 \%$ of the patients had a respiratory focus of infection, while the remainder demonstrated other etiologies. From a total of 30 septic patients, 18 (60\%) required organ replacement therapy and $20(66.67 \%)$ were vasopressor-dependent at inclusion in the study. Our sepsis cohort had a median (IQR) SOFA score of 9 (5-12), indicating moderate disease severity. Nine (30\%) patients were included during the first $24 \mathrm{~h}$ of sepsis. However, this subgroup did not differ from patients recruited thereafter (Additional file 1: Table S2).

The sublingual glycocalyx assessment revealed significantly higher PBR values in septic patients compared to those in healthy controls $(2.04 \mu \mathrm{m}$ [95\% CI 1.97 to 2.1$]$ vs $2.34 \mu \mathrm{m}$ [95\% CI 2.21 to 2.46$], p<0.0001$ ). This difference was present across all three predefined microvascular diameter categories (Fig. 1a). Circulating levels of the glycocalyx core protein syn- 1 were about tenfold higher in septic patients compared to the levels in controls (204.5 $\mathrm{ng} / \mathrm{ml}$ [95\% CI 114.2 to 358.9 ] vs $21.3 \mathrm{ng} / \mathrm{ml}$ [95\% CI 13.2 to 56.7], $p<0.0001)$ and correlated moderately with PBR values ( $\mathrm{rs}=0.51$ [95\% CI 0.22 to 0.72 ], $p=0.0009$; Fig. 1b).

Moreover, we exposed ECs to sterile-filtered, randomly selected sera (5\%; diluted in buffer) from three septic patients and three apparently healthy donors for $24 \mathrm{~h}$. The delta changes of eGC thickness measured in vitro correlated strongly with paired PBR values obtained at the bedside ( $\mathrm{rs}=-0.94, p=0.02$, Fig. $1 \mathrm{c}$ and Additional file 1 : Figure S2).

Analysis of IDF videos showed that among the different parameters of microcirculatory perfusion, the MFI (2.74 points [ $95 \%$ CI 2.58 to 2.91] vs 2.93 points [95\% CI 2.89 to 2.96], $p=0.002)$ and the PPV (92.58\% [95\% CI 85.63 to 97.14 ] vs $98.73 \%$ [95\% CI 96.55 to 99.80], $p=0.0004$ ) were significantly lower in septic patients compared to the controls (Fig. 2a, b). The PVD tended to be lower in septic patients $(16.97 \mathrm{~mm} /$ $\mathrm{mm}^{2}$ [95\% CI 14.96 to 19.87 ] vs $18.54 \mathrm{~mm} / \mathrm{mm}^{2}$ [95\% CI 17.19 to 21.26], $p=0.08$, Fig. 2c), whereas the TVD was no different between the two groups (19.17 $\mathrm{mm} / \mathrm{mm}^{2}$ [95\% CI 17.06 to 20.24 ] vs $18.88 \mathrm{~mm} / \mathrm{mm}^{2}$ [95\% CI 17.56 to 21.68], $p=0.87$ ). Neither PBR nor MFI and PPV were related to age, sex, or comorbidities (Additional file 1: Table S3).

In a pooled analysis of the measurements from all 40 study participants, PBR and microcirculation parameters (MFI and PPV) were correlated with several markers of critical/acute illness (Table 2), including mean arterial pressure (MAP), C-reactive protein (CRP), interleukin-6 (IL-6), and procalcitonin (PCT) levels, as well as SIRS and SOFA scores. Nevertheless, no association between PBR and microcirculatory parameters could be revealed, even after adjusting for important demographic and clinical variables (Additional file 1: Table S4).

Neither PBR values nor syn-1 levels correlated with any of the microcirculatory parameters in the sepsis cohort (Additional file 1: Table S5 and Figure S3). This lack of association persisted even when we dichotomized 

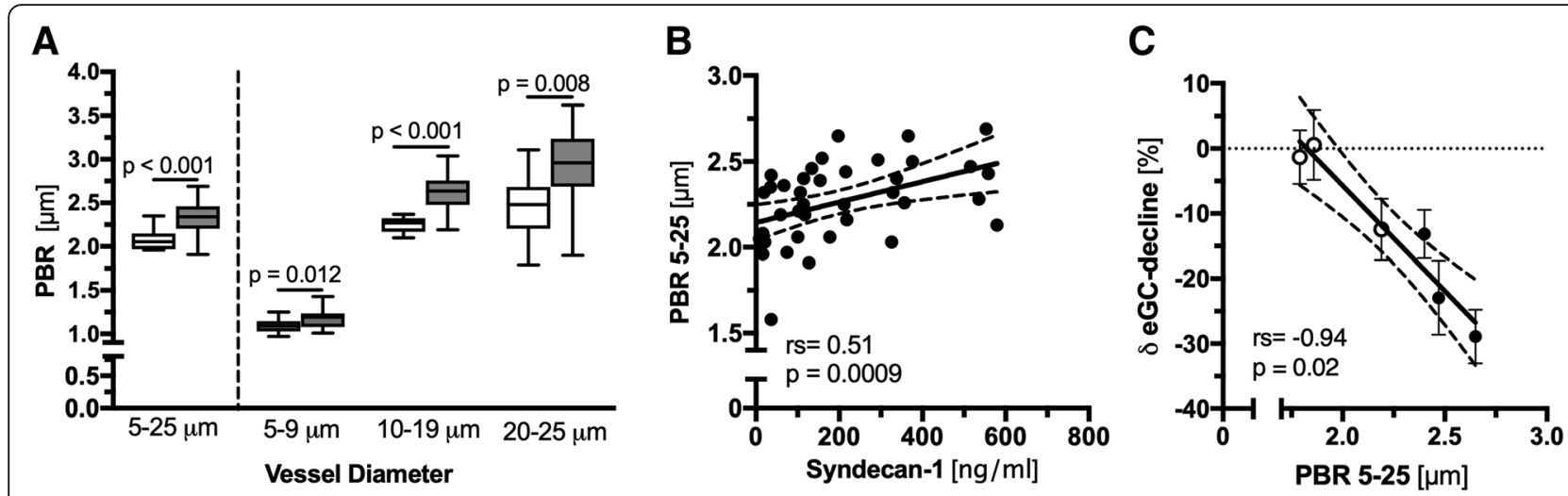

Fig. 1 Endothelial glycocalyx dimensions measured in vivo and in vitro. a Boxplots of PBR values of healthy controls (white) and septic patients (grey) based on the different microvascular diameter ranges. b Correlation of sublingually measured PBR and paired syndecan-1 values. c A subpopulation from a was randomly selected, and ECs were incubated with $5 \%$ sterile-filtered human serum from three septic patients (black circles) and three apparently healthy individuals (white circles), respectively. Scatter plot showing the association between AFM-derived eGC decline (in vitro) and corresponding PBR values (in vivo). Each circle represents the mean of three independent experiments (consisting of $\geq 5$ indentation curves in each of $\geq 10$ cells) for each individual serum. Incubation without human serum served as control. Data are presented as mean \pm SEM. AFM, atomic force microscopy; eGC, endothelial glycocalyx; ICU, intensive care unit; PBR, perfused boundary region; SEM, standard error of mean

the sepsis group by the median PBR value (Fig. $3 \mathrm{a}-\mathrm{c})$. In a different approach, we classified the patients based on their microcirculation parameters into the following three groups: "intact" (PPV $>90$ and MFI $>2.9$ ), "at risk" $(\mathrm{PPV} \leq 90$ or $\mathrm{MFI} \leq 2.9$ ), and "impaired" (PPV $\leq 90$ and MFI $\leq 2.9$ ). Again, no difference was observed between the groups regarding PBR, syn-1 levels, or MAP (Fig. 3d-f).

\section{Discussion}

To the best of our knowledge, this is the first study specifically investigating the associations between the eGC dimensions and established parameters of the sublingual microcirculation in patients with sepsis. The main results of the current study revealed that, although both microcirculation and glycocalyx parameters correlated plausibly with markers of acute/critical illness, no association could be established between the eGC thickness and the microcirculation data obtained (TVD, PVD, PPV, MFI, HI). Furthermore, we investigated, for the first time, the accuracy of the calculated in vivo PBR. This correlated well with circulating syn-1, a marker for glycocalyx shedding, and was in good agreement with glycocalyx thickness measured by AFM, a method that can detect even the modest changes in the nanomechanics of the eGC [37].

The observed dissociation between PBR dimensions and microvascular perfusion in sepsis has already been indicated by two earlier interventional studies examining the effects of leuko-depleted RBC transfusions or
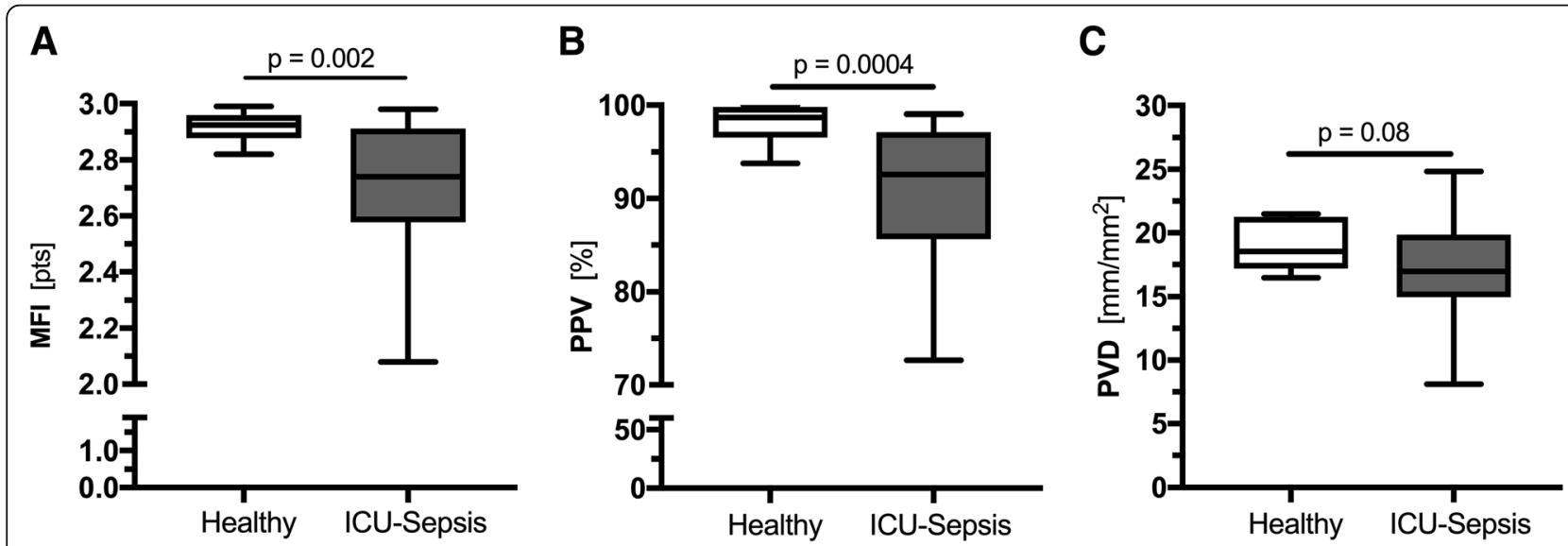

Fig. 2 Boxplots of microcirculation parameters in healthy controls and septic patients. a MFI and b PPV values revealed a damaged microcirculation in the ICU septic population. c The measured PVD tended to be lower in septic patients compared to the healthy controls. ICU, intensive care unit; MFI, microvascular flow index; PPV, proportion of perfused vessels; PVD, perfused vessel density 
Table 2 Significant correlations of PBR, MFI, and PPV with laboratory data and acute/critical illness scores

\begin{tabular}{llll}
\hline Variable & PBR & MFI & PPV \\
\hline CCl (points) & $0.39(0.08 \text { to } 0.63)^{*}$ & - & - \\
CRP $(\mathrm{mg} / \mathrm{dl})$ & $0.54(0.26 \text { to } 0.74)^{* * *}$ & $-0.59(-0.76 \text { to }-0.32)^{* * *}$ & $-0.61(-0.78 \text { to }-0.35)^{* * *}$ \\
Heart rate $($ pulse/min) & - & $-0.41(-0.64 \text { to }-0.10)^{* *}$ & $-0.46(-0.68 \text { to }-0.16)^{* *}$ \\
IL-6 $(\mathrm{ng} / \mathrm{ml})$ & $0.45(0.14 \text { to } 0.68)^{* *}$ & - & - \\
MAP $(\mathrm{mmHg})$ & $-0.37(-0.62 \text { to }-0.06)^{*}$ & - & $-33(0.01 \text { to } 0.58)^{*}$ \\
PCT (ng/ml) & $0.37(0.37 \text { to } 0.62)^{*}$ & - & $-0.41(-0.65 \text { to }-0.11)^{* *}$ \\
SIRS score (points) & $0.44(0.14 \text { to } 0.67)^{* *}$ & - & - \\
SOFA score (points) & $0.44(0.14 \text { to } 0.67)^{* *}$ & $0.53(0.23 \text { to } 0.73)^{* * *}$ & $0.50(0.20 \text { to } 0.72)^{* *}$ \\
Total serum protein $(\mathrm{g} / \mathrm{dl})$ & $-0.39(-0.64 \text { to }-0.06)^{*}$ & &
\end{tabular}

Spearman correlation was used

CRP C-reactive protein, IL-6 interleukin-6, MAP mean arterial pressure, MFI microvascular flow index, PBR perfused boundary region 5-25 $\mu \mathrm{m}, P C T$ procalcitonin, $P P V$ proportion of perfused vessels, SOFA score Sequential Organ Failure Assessment score

${ }^{*} p<0.05,{ }^{* *} p<0.01,{ }^{* * *} p<0.001$

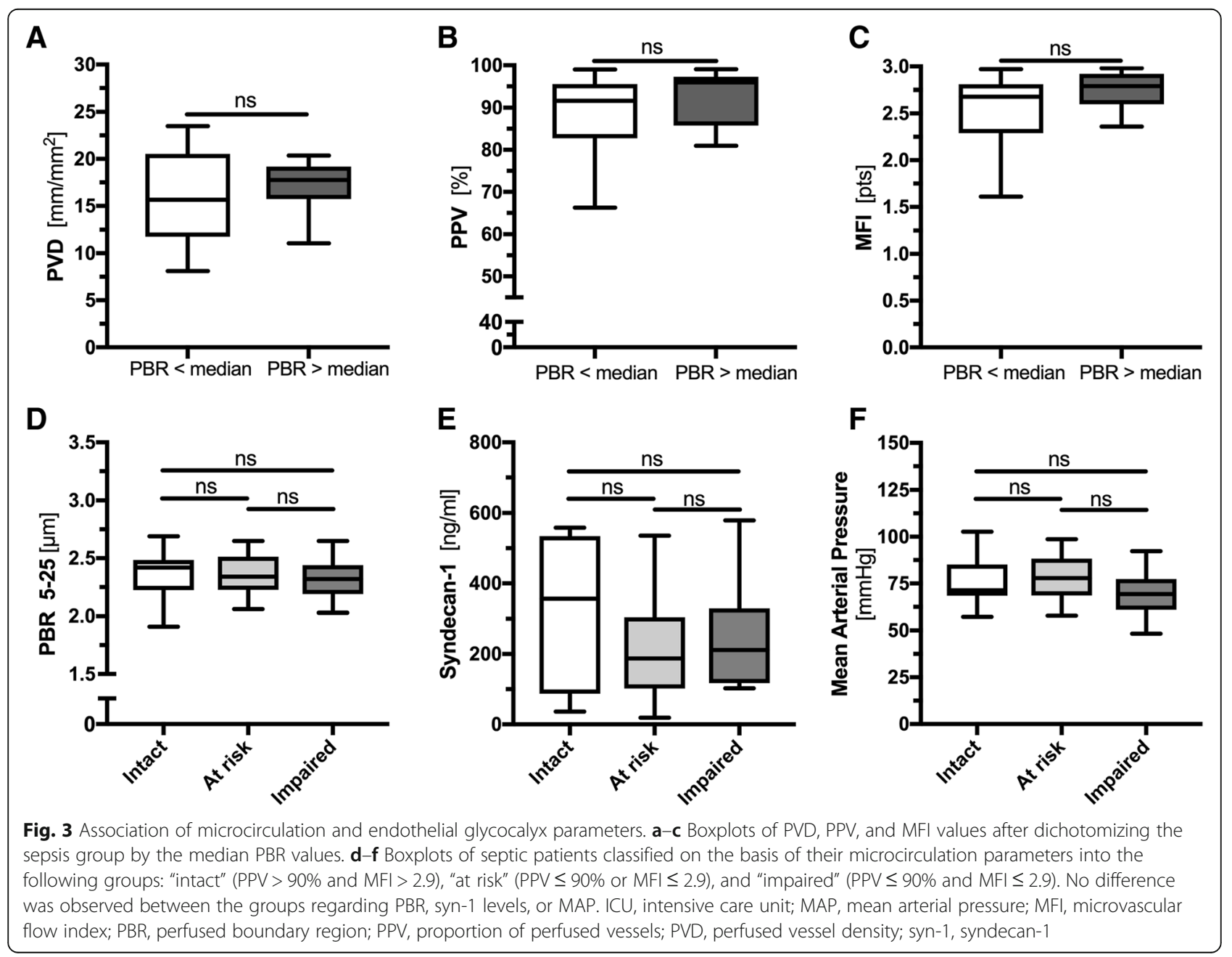


activated protein C, respectively $[19,20]$. In both studies, median PBR values remained unchanged while an improvement in microvascular parameters was seen in the treatment groups. In contrast, two longitudinal studies conducted in patients undergoing cardiac surgery suggested the existence of an association between the alterations in microcirculation parameters and eGC dimensions [18, 41]: Koning et al. assessed the sublingual microcirculation and eGC dimensions perioperatively in 36 patients undergoing cardiac surgery with or without cardiopulmonary bypass (CPB) [18]. The CPB procedure increased the PBR and decreased the PVD, whereas offpump surgery decreased the PBR without affecting the PVD. Dekker et al. analyzed the sublingual microvasculature in 17 patients undergoing non-pulsatile CPB [41]. They found a small increase in PBR and a sustained decrease in PVD and PPV during the 72-h follow-up. Although PBR values during CPB correlated moderately with shed levels of the glycosaminoglycan heparan sulfate, another major component of the glycocalyx, no correlation between PBR and classical parameters of the microcirculation was found. It appears that the reported association between the eGC dimensions or shedding products and microcirculation parameters reflects CPB-induced concordant longitudinal changes, rather than true correlations between the parameters at single time points.

Clinical studies monitoring the sublingual microcirculation during resuscitation procedures revealed the existence of a dissociation between micro- and macrocirculation in septic patients [28, 42]. This phenomenon was recently termed "loss of hemodynamic coherence" [42-45]. In line with these studies, we did not observe any correlation of either SOFA score or MAP with MFI. It is intriguing, however, that this dissociation seems to be absent for the eGC, since eGC dimensions did correlate plausibly with parameters of disease severity and macrocirculation. Our results strengthen the notion that microvascular perfusion and eGC dimensions are two differentially regulated entities, which do not inevitably show concordant changes. In other words, not all patients with an impaired microcirculation exhibit a damaged eGC, and vice versa. The challenging question is why is that? It is conceivable that several factors play a role.

First, we have learned from preclinical murine studies that pulmonary glycocalyx loss develops within $8 \mathrm{~h}$ after the induction of polymicrobial abdominal sepsis [46], whereas injection of Escherichia coli lipopolysaccharides (LPS) results in overt glycocalyx damage within $30 \mathrm{~min}$ [9]. In human endotoxemia, sublingual eGC thickness was lower at $4 \mathrm{~h}$ after LPS administration [47]. In summary, a likely explanation would be that the threshold for, as well as the onset of, eGC damage in human sepsis is diverse, leading to spatiotemporal uncoupling of the two entities.

Second, previous studies have shown that, despite the loss of hemodynamic coherence in individual patients, the classical parameters of sepsis-induced microcirculatory dysfunction respond to resuscitation and optimization of arterial pressure in general $[24,28,48]$. Consistent with this notion, De Backer et al. reported that microcirculatory alterations are less severe in the later phase of sepsis than in the earlier phase [28]. Microcirculatory and PBR derangements in our resuscitated sepsis cohort (only $10 \%$ in shock) were comparable to values in ICU patients with moderate disease severity reported by other groups [19, 21, 27, 49]. Data regarding the regeneration of the eGC in both human and murine sepsis are missing. Cell culture experiments indicate that after enzymatic degradation, full recovery of the eGC requires at least 3 days under normal (i.e., non-septic) in vitro conditions [50, 51]. Although extrapolation of this finding to the ICU is not valid, it is conceivable that regeneration of the eGC in our patients may considerably lag behind the successful stabilization of the macro- and microcirculation. What makes it even more complicated is the possibility that targeted interventions intended at improving the macrocirculation-for example, the correction of hypovolemia [26] - may augment or even induce glycocalyx degradation, if performed excessively $[8,52]$.

Third, another explanation could be that the two entities, microcirculation and eGC, are controlled by different regulatory and compensatory mechanisms, including hormonal, neural, biochemical, and vascular control systems [53-56]. We have recently shown, for example, that the activational state of the endothelialspecific Tie2 receptor controls glycocalyx damage in a non-redundant fashion [39]. However, the Tie2 receptor is not expressed by vascular smooth muscle cells and has no direct effect on vasomotor tone.

We acknowledge some limitations in our study. First, it is a single-center study with a limited sample size. Nevertheless, we could not detect any trend pointing to an association between classical microcirculation parameters and eGC dimensions. The reason for the small number of serum samples used for the in vitro experiments is that the AFM technique is time-consuming and sophisticated, which precludes the analysis of a considerably larger random sample. Further, it is possible that our AFM approach, in the absence of fluid shear stress and/or reduced abundance of plasma proteins, can only detect thickness in denser glycocalyx layers close to the plasma membrane, thereby potentially underestimating the "fluffier" outer (bloodstream-oriented) regions of the eGC $[57,58]$. Second, we had to use two separate camera systems to visualize the sublingual microvasculature and 
thus cannot exclude a sampling error. However, the fact that the real-time assessment of MFI by eyeballing showed good agreement between the two subsequent videomicroscopic examinations (Additional file 1: Figure S4) argues against this hypothesis. Third, we cannot exclude that the use of two different techniques (SDF, IDF) might contribute to the observed dissociation. IDF has a better definition and magnification and can detect a larger number of vessels than SDF. However, as the GlycoCheck ${ }^{\mathrm{mt}}$ system can reliably detect and analyze RBC flux (and thus calculate the PBR) even in the capillaries down to a size of $4 \mu \mathrm{m}$, this difference is probably negligible. Furthermore, the absence of any correlation between microcirculatory and eGC parameters (both PBR and syndecan-1) supports our results. Fourth, our study had a cross-sectional design and was set up neither to detect causality of eGC or microcirculation alterations nor to test the performance of PBR and microcirculation parameters for the outcome prediction. To clarify these issues, we initiated two prospective, observational, longitudinal studies to evaluate the eGC dimensions together with microcirculation analysis in the ER (Early Detection of $\mathrm{x}$ Damage in Emergency Room Patients-the EDGE Study, Clinicaltrials.gov Identifier: NCT03126032) and in the ICU (Analysis of Sublingual Glycocalyx Damage at ICU Admission to Predict Risk of Death-the ASGARD Study, Clinical trials.gov Identifier: NCT03847493).

\section{Conclusions and outlook}

In summary, our data indicate an uncoupling between eGC and microcirculatory parameters in resuscitated sepsis. The mechanism behind this observed dissociation remains unclear. Therefore, future experimental and clinical studies are needed to unravel the relationship of glycocalyx damage and microvascular impairment, as well as their prognostic and therapeutic importance in sepsis.

\section{Additional file}

\section{Additional file 1: Table S1. Summary of microcirculation and} endothelial glycocalyx parameters $[16,25]$. Figure S1. Analysis of the endothelial glycocalyx (eGC) in cell culture via atomic force microscopy (AFM). Surface approach: the cantilever (AFM tip) approaches the sample surface vertically. The reflection of a laser beam from the back of the cantilever is continuously detected by a photodiode. First slope: reaching the surface, the cantilever, serving as a soft spring, is deflected while indenting into the sample. The changing laser beam reflection is plotted as a function of sample position along the $z$-axis. By including the cantilever's spring constant and the optical lever sensitivity, a forceversus-indentation curve can be generated to provide information about how much force (in pN) is needed to indent a certain distance (in $\mathrm{nm}$ ) into the sample. The first slope of the curve reflects the indentation of the eGC. Second slope: in the second slope, more force is needed to indent into the surface, which reflects the cell cortex with the plasma membrane and actin web. Due to the linearity of the first slope, a regression line can be generated manually through the starting points of both slopes using PUNIAS (protein unfolding and nanoindentation analysis software, version 1.0, release 2.1, http://punias. free.fr). Projected to the $x$-axis, the distance between both starting points represents the thickness of the eGC $[38,39]$. Table S2. Baseline characteristics of septic patients stratified for sepsis duration. Figure S2. Endothelial glycocalyx dimensions measured in vivo and in vitro. Scatter plot showing the association between AFM-derived eGC thickness (in vitro) and corresponding PBR values (in vivo) in three apparently healthy individuals (white circles) and in three septic patients (black circles). Each circle represents the mean of three independent experiments (consisting of $\geq 5$ indentation curves in each of $\geq 10$ cells) for each individual serum. Incubation without human serum served as control. Data are presented as mean \pm SEM. Table S3. Correlations of PBR, PPV, MFI, age, sex, and comorbidities in the septic cohort. Spearman correlation was used. The $p$ values are indicated in brackets. Table $\mathbf{S} 4$. Simple and multiple linear regression (PBR as dependent variable). Dependent variable: PBR. Table S5. Correlations of microcirculatory and eGC parameters in the septic cohort. Spearman correlation was used. The $p$ values are indicated in brackets. Figure S3. Correlations of microcirculation and endothelial glycocalyx parameters. (A-C): Correlation of PBR with PVD, PPV, and MFI. (D-F): Correlation of syndecan-1 with PVD, PPV, and MFI. Figure S4. MFI (eyeballing) obtained in real-time with the two different systems. (A): Correlation between the MFI (eyeballing) values obtained at the bedside. (B): Bland-Altman plot showing the limits of agreement (bias $\pm 1.96 \mathrm{SD}$ ) between paired MFI values for the Cytocam and GlycoCheck $\mathrm{K}^{\mathrm{T}}$ system (eyeballing). One point can represent more than one individual. (DOCX 606 kb)

\section{Abbreviations}

AFM: Atomic force microscopy/microscope; BMI: Body mass index; CCl: Charlson Comorbidity Index; CKD: Chronic kidney disease; CNS: Central nervous system; CPB: Cardiopulmonary bypass; CRP: C-reactive protein; EC: Endothelial cell; eGC: Endothelial glycocalyx; ELISA: Enzyme-linked immunosorbent assay; ER: Emergency room; FBS: Fetal bovine serum; HI: Heterogeneity index; ICU: Intensive care unit; IDF: Incident dark field; IL-6: Interleukin-6; IQR: Interquartile range; LPS: Lipopolysaccharides; MAP: Mean arterial pressure; MFI: Microvascular flow index; PBR: Perfused boundary region; PCT: Procalcitonin; PPV: Proportion of perfused vessels; PVD: Perfused vessel density; RBC: Red blood cell; RBCW: Red blood cell column width; SD: Standard deviation; SDF: Sidestream dark field; SEM: Standard error of mean; SIRS: Systemic inflammatory response syndrome; SOFA: Sequential Organ Failure Assessment; syn-1: Syndecan-1; TVD: Total vessel density; WBC: White blood cell

\section{Acknowledgements}

We would like to thank our ICU colleagues for supporting our study. We would like to acknowledge the support of the Medizinerkolleg Münster - MedK (to TP), the Centre for Clinical Trials Münster (to AR), and the Open Access Publication Fund of the University of Münster.

\section{Authors' contributions}

AR designed the study, recorded the clinical and demographic data, performed the sublingual eGC measurements, analyzed the data, prepared the figures, and drafted the manuscript. LMS contributed to the design of the study, obtained the classical microcirculation parameters, and revised the manuscript. TP performed the AFM experiments. HV, HP, and CE contributed to the design of the study, interpreted the findings, and revised the manuscript. MH contributed to the design of the study, performed the offline analysis of the microcirculation parameters, interpreted the findings, and revised the manuscript. PK had the initial idea, supervised the study, interpreted the findings, and contributed to the manuscript. All authors read and approved the final manuscript.

\section{Funding}

We would like to thank the Faculty of Medicine of the University of Münster for providing partial funding (to AR).

\section{Availability of data and materials}

The datasets used and/or analyzed during the current study are available from the corresponding author on reasonable request. 


\section{Ethics approval and consent to participate}

The study was performed in accordance with the Declaration of Helsinki and approved by the Ethics Committee of the General Medical Council Westfalen-Lippe and the WWU Münster, Germany (file number: 2016-073-f-S). Patients were enrolled after written informed consent was obtained from them or their legal representatives.

\section{Consent for publication}

Consent for publication has been obtained.

\section{Competing interests}

$A R, L M S, T P, H P, C E, M H$, and PK declare that they have no competing interests. HV is a Chief Science Officer of GlycoCheck BV, The Netherlands.

\section{Author details}

'Department of Medicine D, Division of General Internal Medicine, Nephrology, and Rheumatology, University Hospital Muenster, Albert-Schweitzer-Campus 1, 48149 Münster, Germany. ${ }^{2}$ Department of Anesthesiology, Intensive Care, and Pain Therapy, University Hospital Muenster, Münster, Germany. ${ }^{3}$ Department of Physiology, Cardiovascular Research Institute Maastricht, Maastricht University, Maastricht, The Netherlands.

Received: 26 March 2019 Accepted: 16 July 2019

\section{Published online: 24 July 2019}

\section{References}

1. Reitsma S, Slaaf DW, Vink H, van Zandvoort MA, oude Egbrink MG. The endothelial glycocalyx: composition, functions, and visualization. Pflugers Arch. 2007;454(3):345-59.

2. van Haaren PMA, VanBavel E, Vink H, Spaan JAE. Localization of the permeability barrier to solutes in isolated arteries by confocal microscopy. Am J Physiol Heart Circ Physiol. 2003;285(6):H2848-56.

3. Donati A, Damiani E, Domizi R, Romano R, Adrario E, Pelaia P, Ince C, Singer M. Alteration of the sublingual microvascular glycocalyx in critically ill patients. Microvasc Res. 2013;90:86-9.

4. Alphonsus CS, Rodseth RN. The endothelial glycocalyx: a review of the vascular barrier. Anaesthesia. 2014;69(7):777-84.

5. Mulivor AW, Lipowsky HH. Role of glycocalyx in leukocyte-endothelial cell adhesion. Am J Physiol Heart Circ Physiol. 2002;283(4):H1282-91.

6. Curry FE, Adamson RH. Endothelial glycocalyx: permeability barrier and mechanosensor. Ann Biomed Eng. 2012;40(4):828-39.

7. Salmon AH, Ferguson JK, Burford JL, Gevorgyan H, Nakano D, Harper SJ, Bates DO, Peti-Peterdi J. Loss of the endothelial glycocalyx links albuminuria and vascular dysfunction. J Am Soc Nephrol. 2012;23(8):1339-50.

8. Uchimido R, Schmidt EP, Shapiro NI. The glycocalyx: a novel diagnostic and therapeutic target in sepsis. Crit Care. 2019;23(1):16.

9. Schmidt EP, Yang $Y$, Janssen WJ, Gandjeva A, Perez MJ, Barthel L, Zemans RL, Bowman JC, Koyanagi DE, Yunt ZX, et al. The pulmonary endothelial glycocalyx regulates neutrophil adhesion and lung injury during experimental sepsis. Nat Med. 2012;18(8):1217-23.

10. Padberg JS, Wiesinger A, di Marco GS, Reuter S, Grabner A, Kentrup D, Lukasz A, Oberleithner H, Pavenstadt H, Brand M, et al. Damage of the endothelial glycocalyx in chronic kidney disease. Atherosclerosis. 2014; 234(2):335-43.

11. Ostrowski SR, Haase N, Muller RB, Moller MH, Pott FC, Perner A, Johansson $\mathrm{PI}$. Association between biomarkers of endothelial injury and hypocoagulability in patients with severe sepsis: a prospective study. Crit Care. 2015;19:191.

12. Ostrowski SR, Pedersen SH, Jensen JS, Mogelvang R, Johansson PI. Acute myocardial infarction is associated with endothelial glycocalyx and cell damage and a parallel increase in circulating catecholamines. Crit Care. 2013;17(1):R32

13. Nelson A, Berkestedt I, Schmidtchen A, Ljunggren L, Bodelsson M. Increased levels of glycosaminoglycans during septic shock: relation to mortality and the antibacterial actions of plasma. Shock. 2008;30(6):623-7.

14. Anand D, Ray S, Srivastava LM, Bhargava S. Evolution of serum hyaluronan and syndecan levels in prognosis of sepsis patients. Clin Biochem. 2016: 49(10-11):768-76.

15. Puskarich MA, Cornelius DC, Tharp J, Nandi U, Jones AE. Plasma syndecan-1 levels identify a cohort of patients with severe sepsis at high risk for intubation after large-volume intravenous fluid resuscitation. J Crit Care. 2016;36:125-9.

16. Lee DH, Dane MJ, van den Berg BM, Boels MG, van Teeffelen JW, de Mutsert R, den Heijer M, Rosendaal FR, van der Vlag J, van Zonneveld AJ, et al. Deeper penetration of erythrocytes into the endothelial glycocalyx is associated with impaired microvascular perfusion. PLoS One. 2014;9(5):e96477.

17. Martens RJ, Vink H, van Oostenbrugge RJ, Staals J. Sublingual microvascular glycocalyx dimensions in lacunar stroke patients. Cerebrovasc Dis. 2013:35(5):451-4.

18. Koning $\mathrm{NJ}$, Vonk $A B$, Vink $H$, Boer C. Side-by-side alterations in glycocalyx thickness and perfused microvascular density during acute microcirculatory alterations in cardiac surgery. Microcirculation. 2016;23(1):69-74.

19. Donati A, Damiani E, Luchetti M, Domizi R, Scorcella C, Carsetti A, Gabbanelli V, Carletti P, Bencivenga R, Vink H, et al. Microcirculatory effects of the transfusion of leukodepleted or non-leukodepleted red blood cells in patients with sepsis: a pilot study. Crit Care. 2014;18(1):R33.

20. Donati A, Damiani E, Botticelli L, Adrario E, Lombrano MR, Domizi R, Marini B, Van Teeffelen JW, Carletti P, Girardis M, et al. The aPC treatment improves microcirculation in severe sepsis/septic shock syndrome. BMC Anesthesiol. 2013;13(1):25.

21. Rovas A, Lukasz AH, Vink H, Urban M, Sackarnd J, Pavenstadt H, Kumpers P. Bedside analysis of the sublingual microvascular glycocalyx in the emergency room and intensive care unit - the GlycoNurse study. Scand J Trauma Resusc Emerg Med. 2018;26(1):16.

22. Ince $\mathrm{C}$. The rationale for microcirculatory guided fluid therapy. Curr Opin Crit Care. 2014;20(3):301-8.

23. Ince C, Boerma EC, Cecconi M, De Backer D, Shapiro NI, Duranteau J, Pinsky MR, Artigas A, Teboul JL, Reiss IKM, et al. Second consensus on the assessment of sublingual microcirculation in critically ill patients: results from a task force of the European Society of Intensive Care Medicine. Intensive Care Med. 2018:44(3):281-99.

24. Xu JY, Ma SQ, Pan C, He HL, Cai SX, Hu SL, Liu AR, Liu L, Huang YZ, Guo FM, et al. A high mean arterial pressure target is associated with improved microcirculation in septic shock patients with previous hypertension: a prospective open label study. Crit Care. 2015;19:130.

25. De Backer D, Hollenberg S, Boerma C, Goedhart P, Buchele G, OspinaTascon G, Dobbe I, Ince C. How to evaluate the microcirculation: report of a round table conference. Crit Care. 2007;11(5):R101.

26. Pranskunas A, Koopmans M, Koetsier PM, Pilvinis V, Boerma EC Microcirculatory blood flow as a tool to select ICU patients eligible for fluid therapy. Intensive Care Med. 2013;39(4):612-9.

27. Massey MJ, Hou PC, Filbin M, Wang H, Ngo L, Huang DT, Aird WC, Novack $V$, Trzeciak S, Yealy DM, et al. Microcirculatory perfusion disturbances in septic shock: results from the ProCESS trial. Crit Care. 2018;22(1):308.

28. De Backer D, Donadello K, Sakr Y, Ospina-Tascon G, Salgado D, Scolletta S, Vincent JL. Microcirculatory alterations in patients with severe sepsis: impact of time of assessment and relationship with outcome. Crit Care Med. 2013; 41(3):791-9.

29. Singer M, Deutschman CS, Seymour CW, Shankar-Hari M, Annane D, Bauer M, Bellomo R, Bernard GR, Chiche JD, Coopersmith CM, et al. The Third International Consensus Definitions for Sepsis and Septic Shock (Sepsis-3). JAMA. 2016;315(8):801-10.

30. Quan H, Li B, Couris CM, Fushimi K, Graham P, Hider P, Januel JM, Sundararajan V. Updating and validating the Charlson comorbidity index and score for risk adjustment in hospital discharge abstracts using data from 6 countries. Am J Epidemiol. 2011;173(6):676-82.

31. Tanaka S, Harrois A, Nicolai C, Flores M, Hamada S, Vicaut E, Duranteau J. Qualitative real-time analysis by nurses of sublingual microcirculation in intensive care unit: the MICRONURSE study. Crit Care. 2015;19:388.

32. Hutchings S, Watts S, Kirkman E. The Cytocam video microscope. A new method for visualising the microcirculation using incident dark field technology. Clin Hemorheol Microcirc. 2016;62(3):261-71.

33. Massey MJ, Larochelle E, Najarro G, Karmacharla A, Arnold R, Trzeciak S, Angus DC, Shapiro NI. The microcirculation image quality score: development and preliminary evaluation of a proposed approach to grading quality of image acquisition for bedside videomicroscopy. J Crit Care. 2013;28(6):913-7.

34. Dobbe JG, Streekstra GJ, Atasever B, van Zijderveld R, Ince C. Measurement of functional microcirculatory geometry and velocity distributions using automated image analysis. Med Biol Eng Comput. 2008;46(7):659-70. 
35. Hessler M, Arnemann PH, Zamit F, Seidel L, Kampmeier TG, Kathofer U, Morelli A, Ince C, Rehberg SW, Ertmer C. A new complimentary web-based tool for manual analysis of microcirculation videos: validation of the Capillary Mapper against the current gold standard AVA 3.2. Microcirculation. 2018;25(8):e12505.

36. Massey MJ, Shapiro NI. A guide to human in vivo microcirculatory flow image analysis. Crit Care. 2016;20:35

37. Wiesinger A, Peters W, Chappell D, Kentrup D, Reuter S, Pavenstadt H, Oberleithner $H$, Kumpers P. Nanomechanics of the endothelial glycocalyx in experimental sepsis. PLoS One. 2013;8(11):e80905.

38. Oberleithner $H$, Peters W, Kusche-Vihrog K, Korte S, Schillers H, Kliche K, Oberleithner K. Salt overload damages the glycocalyx sodium barrier of vascular endothelium. Pflugers Arch. 2011;462(4):519-28.

39. Lukasz A, Hillgruber C, Oberleithner H, Kusche-Vihrog K, Pavenstadt H, Rovas A, Hesse B, Goerge T, Kumpers P. Endothelial glycocalyx breakdown is mediated by angiopoietin-2. Cardiovasc Res. 2017;113(6):671-80.

40. Hulley SBCS, Browner WS, Grady D, Newman TB. Designing clinical research: an epidemiologic approach. 4th ed. Philadelphia: Lippincott Williams \& Wilkins; 2013: Appendix 6C. p. 79.

41. Dekker NAM, Veerhoek D, Koning NJ, van Leeuwen ALI, Elbers PWG, van den Brom CE, Vonk ABA, Boer C. Postoperative microcirculatory perfusion and endothelial glycocalyx shedding following cardiac surgery with cardiopulmonary bypass. Anaesthesia. 2019;74(5):609-18.

42. Edul VS, Enrico C, Laviolle B, Vazquez AR, Ince C, Dubin A. Quantitative assessment of the microcirculation in healthy volunteers and in patients with septic shock. Crit Care Med. 2012;40(5):1443-8.

43. Ince $\mathrm{C}$. Hemodynamic coherence and the rationale for monitoring the microcirculation. Crit Care. 2015;19(Suppl 3):S8.

44. Sakr Y, Dubois MJ, De Backer D, Creteur J, Vincent JL. Persistent microcirculatory alterations are associated with organ failure and death in patients with septic shock. Crit Care Med. 2004;32(9):1825-31.

45. Trzeciak S, McCoy JV, Phillip Dellinger R, Arnold RC, Rizzuto M, Abate NL, Shapiro NI, Parrillo JE, Hollenberg SM, Microcirculatory Alterations in R, et al. Early increases in microcirculatory perfusion during protocol-directed resuscitation are associated with reduced multi-organ failure at $24 \mathrm{~h}$ in patients with sepsis. Intensive Care Med. 2008;34(12):2210-7.

46. Margraf A, Herter JM, Kuhne K, Stadtmann A, Ermert T, Wenk M, Meersch M, Van Aken H, Zarbock A, Rossaint J. 6\% hydroxyethyl starch (HES 130/0.4) diminishes glycocalyx degradation and decreases vascular permeability during systemic and pulmonary inflammation in mice. Crit Care. 2018;22(1):111

47. Nieuwdorp M, Meuwese MC, Mooij HL, van Lieshout MH, Hayden A, Levi M, Meijers JC, Ince C, Kastelein JJ, Vink H, et al. Tumor necrosis factor-alpha inhibition protects against endotoxin-induced endothelial glycocalyx perturbation. Atherosclerosis. 2009;202(1):296-303.

48. Thooft A, Favory R, Salgado DR, Taccone FS, Donadello K, De Backer D, Creteur J, Vincent JL. Effects of changes in arterial pressure on organ perfusion during septic shock. Crit Care. 2011;15(5):R222.

49. Pranskunas A, Tamosuitis T, Balciuniene N, Damanskyte D, Sneider E, Vitkauskiene A, Sirvinskas E, Pilvinis V, Boerma EC. Alterations of conjunctival glycocalyx and microcirculation in non-septic critically ill patients. Microvasc Res. 2018;118:44-8.

50. Giantsos-Adams KM, Koo AJ, Song S, Sakai J, Sankaran J, Shin JH, GarciaCardena G, Dewey CF Jr. Heparan sulfate regrowth profiles under laminar shear flow following enzymatic degradation. Cell Mol Bioeng. 2013;6(2):160-74.

51. Koo A, Dewey CF Jr, Garcia-Cardena G. Hemodynamic shear stress characteristic of atherosclerosis-resistant regions promotes glycocalyx formation in cultured endothelial cells. Am J Physiol Cell Physiol. 2013; 304(2):C137-46.

52. Iba T, Levy JH. Derangement of the endothelial glycocalyx in sepsis. J Thromb Haemost. 2019;17(2):283-94.

53. Bezemer R, Bartels SA, Bakker J, Ince C. Clinical review: clinical imaging of the sublingual microcirculation in the critically ill--where do we stand? Crit Care. 2012;16(3):224

54. Lelubre C, Vincent JL. Mechanisms and treatment of organ failure in sepsis. Nat Rev Nephrol. 2018;14(7):417-27.

55. De Backer D, Donadello K, Taccone FS, Ospina-Tascon G, Salgado D, Vincent JL. Microcirculatory alterations: potential mechanisms and implications for therapy. Ann Intensive Care. 2011;1(1):27.

56. De Backer D, Orbegozo Cortes D, Donadello K, Vincent JL. Pathophysiology of microcirculatory dysfunction and the pathogenesis of septic shock. Virulence. 2014;5(1):73-9.
57. Chappell D, Jacob M, Paul O, Rehm M, Welsch U, Stoeckelhuber M, Conzen $\mathrm{P}$, Becker BF. The glycocalyx of the human umbilical vein endothelial cell: an impressive structure ex vivo but not in culture. Circ Res. 2009;104(11):1313-7.

58. Bai K, Wang W. Spatio-temporal development of the endothelial glycocalyx layer and its mechanical property in vitro. J R Soc Interface. 2012;9(74):2290-8.

\section{Publisher's Note}

Springer Nature remains neutral with regard to jurisdictional claims in published maps and institutional affiliations.
Ready to submit your research? Choose BMC and benefit from:

- fast, convenient online submission

- thorough peer review by experienced researchers in your field

- rapid publication on acceptance

- support for research data, including large and complex data types

- gold Open Access which fosters wider collaboration and increased citations

- maximum visibility for your research: over $100 \mathrm{M}$ website views per year

At BMC, research is always in progress.

Learn more biomedcentral.com/submissions 